\title{
Preliminary Investigations into the Purification and Functionalization of Multiwall Carbon Nanotubes
}

\author{
G. Trykowski ${ }^{a, *}$, S. Biniak ${ }^{a}$, L. StOBinski $^{b, c}$ And B. LeSiaK ${ }^{c}$ \\ ${ }^{a}$ Department of Chemistry, Nicolaus Copernicus University, Gagarina 7, 87-100 Torun, Poland \\ ${ }^{b}$ Department of Materials Engineering, Warsaw University of Technology, Wołoska 141, 02-507 Warsaw, Poland \\ ${ }^{c}$ Institute of Physical Chemistry, Polish Academy of Sciences, Kasprzaka 44/52, 01-224 Warsaw, Poland \\ The purification and functionalization of commercial multiwall carbon nanotubes was investigated. Carbon \\ nanotubes (CNT CO., Ltd, Korea) were treated with boiling concentrated $\mathrm{HNO}_{3}$ under a reflux condenser for \\ about $50 \mathrm{~h}$ at $120^{\circ} \mathrm{C}$ in order to purify and oxidize the raw material. The oxidized multiwall carbon nanotubes \\ were rinsed with deionized water until stabilization of the filtrate $\mathrm{pH}$. Measurement techniques included elemental \\ analysis (CHN), scanning electron microscopy with energy dispersive X-ray spectrometer, inductively coupled \\ plasma mass spectrometry, Fourier transform infrared spectroscopy and thermal analysis. With the measurement \\ techniques used the following information was obtained: CHN analysis provided information about the quantitative \\ composition of the following elements carbon, hydrogen, nitrogen, scanning electron microscopy imaging provided \\ information on shape, thickness and length of the nanotubes, energy dispersive X-ray spectrometry analysis of \\ information about surface atomic composition of the quantitative analysis, inductively coupled plasma mass \\ spectrometry quantitative analysis of the atomic composition (metals, especially $\mathrm{Fe}, \mathrm{Al}$ ), the Fourier transform \\ infrared studies provided information about qualitative analysis of surface functional groups $\mathrm{C}_{x} \mathrm{O}_{y} \mathrm{H}_{z}(\mathrm{COOH}, \mathrm{OH}$, \\ $\mathrm{COO})$ and thermal gravimetric-differential thermal analysis - quantitative analysis of thermal decomposition \\ products. It was found that oxidation leads to the removal of amorphous carbon and forms mainly carboxylic \\ functional groups linked to the nanotubes. The Fourier transform infrared spectra indicate the presence of some \\ other structures, like ketone (quinone), acid anhydride, ether and epoxy groups. Nitric acid treatment also \\ effectively removes aluminum oxide catalyst and iron catalyst from commercial multiwall carbon nanotubes.
}

PACS numbers: 61.48.De, 81.16.Be, 07.57.Ty, 82.80-d, 68.37.Hk

\section{Introduction}

Carbon nanotubes (CNTs) take a variety of forms - single wall (SWCNTs), double wall (DWCNTs) and multi-wall (MWCNTs) - where the cylindrical concentric planes interact with each other due to the van der Waals forces. Nanotubes and their modified structures are characterized by the following properties: real density similar to that of a polymer, electrical conductivity better than that of copper, excellent flexibility and elasticity, excellent chemical stability, both of metal and semiconductor, a high aspect ratio (50-1000), mechanical strength better than that of steel, excellent thermal conductivity, excellent electron emission $[1,2]$. These parameters make carbon nanotubes a very attractive material for numerous applications. CNTs are starting to be used in nanotechnology, nanoelectronics, nanooptoelectronics, nanobiotechnology, catalysis and as a sorbent [3-8]. Potential practical applications of CNTs include their use as chemical sensors, field emission materials, catalyst supports, electronic devices, high-sensitivity nanobalances for nanoscopic particles, nanotweezers, reinforcements in high-performance composites and as nanoprobes in metrology and biomedical and chemical investigations,

* corresponding author; e-mail: tryki@chem.umk.pl anodes for lithium ions in batteries, nanoelectronic devices, supercapacitors and hydrogen storage. Although the production and purification of MWCNTs are well known and have been described in detail, their functionalization during oxidation (removal of amorphous phase) does not yet appear to be fully explained. In the present work the wet chemical purification, oxidation and functionalization of multiwall carbon nanotubes were investigated [9-12] with respect to the chemical and structural changes between commercial CNTs and CNTs oxidized with concentrated $\mathrm{HNO}_{3}$ at $120^{\circ} \mathrm{C}$. MWCNTs were studied by elemental analysis $(\mathrm{C}, \mathrm{H}, \mathrm{N})$, scanning electron microscopy (SEM) in conjunction with energy dispersive X-ray spectroscopy (EDS), inductively coupled plasma mass spectrometry (ICP-MS), Fourier transform infrared (FTIR) spectroscopy and thermal gravimetric analysisdifferential thermal analysis (TGA-DTA).

\section{Experimental \\ 2.1. Samples}

Commercial MWCNTs (CNT Co., Ltd, Korea), c-MWCNTs, were refluxed with boiling concentrated $(68 \%) \mathrm{HNO}_{3}$ at $120^{\circ} \mathrm{C}$ for about $50 \mathrm{~h}$ in order to completely remove the amorphous carbon phase, traces of catalysts and their supports, and to oxidize the raw material. The oxidized MWCNTs (ox-MWCNTs) were successively eluted with deionized water, ammonia water, 
dilute $\mathrm{HCl}$ and again with deionized water until the filtrate $\mathrm{pH}$ had stabilized.

\subsection{Elemental analysis}

A Vario Macro apparatus (Elementar Analysensysteme $\mathrm{GmbH}$, Germany) was used for the elemental analysis. Beforehand, the sample was dried at $100^{\circ} \mathrm{C}$ for $12 \mathrm{~h}$ in air and then under vacuum $\left(p=10^{-4} \mathrm{~Pa}\right)$ at $25^{\circ} \mathrm{C}$ for $12 \mathrm{~h}$. The catalytic combustion proceeded at $900^{\circ} \mathrm{C}$ in an oxygen atmosphere. After combustion, the separate components, i.e., $\mathrm{N}_{2}, \mathrm{H}_{2} \mathrm{O}$ and $\mathrm{CO}_{2}$ were quantified: the $\mathrm{H}$ content from $\mathrm{H}_{2} \mathrm{O}$, the $\mathrm{C}$ content from $\mathrm{CO}_{2}$ and $\mathrm{N}$ from $\mathrm{N}_{2}$. The $\mathrm{O}$ content was estimated from the difference between this and the content of the other components (C, H, N and ash).

\section{3. $S E M / E D S$}

A $1430 \mathrm{VP}$ scanning electron microscope (SEM) (LEO Electron Microscopy Ltd, England), equipped with detectors of secondary electrons (SE) and backscattered electrons (BSE), and a Quantax 200 energy dispersive X-ray spectrometer (EDS) with an XFlash 4010 detector (Bruker AXS Microanalysis GmbH, Germany) were used to make surface and subsurface (up to $5 \mu \mathrm{m}$ ) morphology and quantitative measurements at room temperature (RT). The EDS detection limits of the spectrometer: the resolution of the point analysis was from 1 to $5 \mu \mathrm{m}$, the accuracy $s$ from 0.5 to $5 \%$, and the maximum sensitivity of the method $10^{-12} \mathrm{~g}$. The mass of the sample used for analysis was approximately $20 \mathrm{mg}$.$$
\text { 2.4. ICP-MS }
$$

The content of metals ( $\mathrm{Fe}$ and $\mathrm{Al}$ ) in MWCNTs was measured on an Agilent 7500 mass spectrometer (ICP-MS). The following mineralization procedure was applied: $10 \mathrm{mg}$ MWCNTs was added to $8 \mathrm{ml}$ concentrated ultrapure $65 \% \mathrm{HNO}_{3}$ and $2 \mathrm{ml} 30 \%$ hydrogen peroxide, then heated in a microwave oven at $200{ }^{\circ} \mathrm{C}$ for $20 \mathrm{~min}$.

\subsection{FTIR}

The FTIR measurements were performed on a Fourier transform spectrometer (Spectrum 2000, Perkin Elmer) in the $500-4000 \mathrm{~cm}^{-1}$ wave number range using pellets containing approximately $1 \mathrm{mg}$ of sample mixed with $300 \mathrm{mg} \mathrm{KBr}$ (spectrally pure) and compressed $\left(150 \mathrm{~kg} / \mathrm{cm}^{2}\right)$. Two ways of preparing pellets for FTIR analysis were used: in the first, pellets of MWCNTs with $\mathrm{KBr}$ after pressing were heated at temperatures 50 , 100,150 , and $250^{\circ} \mathrm{C}$; in the second, previously heated MWCNTs (at 350, 500, 600, 700, 800 and $900^{\circ} \mathrm{C}$, and vacuum $\left.10^{-3} \mathrm{~Pa}\right)$, were pressed with $\mathrm{KBr}$.

\subsection{TGA-DTA}

Measurements were performed on a Simultaneous TGA-DTA thermoanalyser (Thermal Analysis Company — TA Instruments type SDT 2960). The measurement parameters were: temperature range $-20-800^{\circ} \mathrm{C}$, heating rate $10^{\circ} \mathrm{C} / \mathrm{min}$, sensitivity $0.1 \mathrm{mg}$, inert atmosphere (nitrogen, purity 99.999\%), sample weight ca. $12 \mathrm{mg}$. Before measurement, samples were dried at $110^{\circ} \mathrm{C}$.

\section{Results and discussion}

The effects of purification and functionalization of commercial MWCNTs samples are visible to the naked eye. The c-MWCNTs are solid black and powdery, while the ox-MWCNTs take the form of a black granular substance that can be rolled to a pulp in the fingers. A small quantity $(1 \mathrm{mg})$ of nanotubes dispersed in a polar solvent (20 ml ethanol or water) yields a black slurry, but only ox-MWCNTs remain in colloidal "solution" (stable even after one year); crude c-MWCNTs fall to the bottom of the vessel.

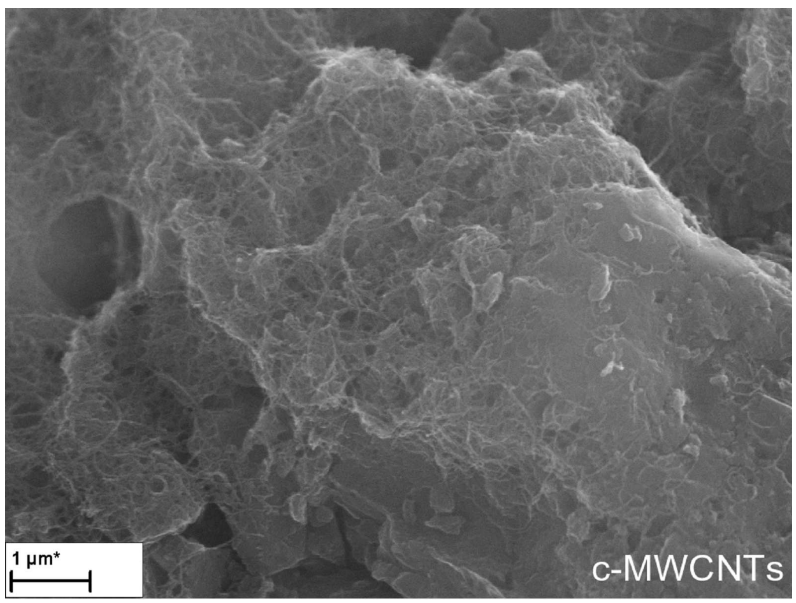

Fig. 1. SEM images of c-MWCNTs supported on active carbon granules.

CHN elemental analysis yielded the quantitative composition of the light elements (carbon, hydrogen, nitrogen and oxygen). There were significant differences in oxygen content between the samples (Table). The oxygen content in the oxidized sample increased by $7 \mathrm{wt} \%$; there was a simultaneous relative decrease in the carbon content. The share of hydrogen and nitrogen remained stable, but was not greater than $1 \mathrm{wt} \%$. EDS supplied information on the complete qualitative composition of the samples, and the results of the quantitative determinations, in particular those of the light elements are overestimated and should be treated as approximate. The surface oxygen content (from EDS analysis) of c-MWCNTs is nearly 2.5 greater than the oxygen in the bulk solid, which suggests spontaneous oxidation of the carbon material on contact with air following its manufacture under vacuum. EDS analysis also shows that the c-MWCNT samples contain significant quantities of iron and aluminum (Table) that are not found in the ox-MWCNT samples. ICP-MS is a good method for quantifying metals, particularly aluminum and iron. ICP-MS analysis indicates that during oxidation, iron is removed completely and only trace 
amounts of aluminum remain. Summarizing the results of quantitative analysis (CHN, EDS and ICP-MS), it can be stated that the oxidation of commercial samples in the presence of $7 \%$ oxygen effectively removed iron and aluminum. The microscopic pattern shows the shape, thickness and length of the commercial nanotubes supported on active carbon granules (Fig. 1). A satisfactory resolution was not achieved with SEM, which helped to differentiate the samples.

Comparison of atomic content.

TABLE

\begin{tabular}{c|c|c|c|c|c|c}
\hline \hline Element & $\begin{array}{c}\text { Elemental } \\
\text { analysis [wt\%] }\end{array}$ & $\begin{array}{c}\text { EDS } \\
{[\mathrm{wt} \%]}\end{array}$ & $\begin{array}{c}\text { ICP-MS } \\
{[\mathrm{wt} \%]}\end{array}$ & $\begin{array}{c}\text { Elemental } \\
\text { analysis [wt\%] }\end{array}$ & $\begin{array}{c}\text { EDS } \\
{[\mathrm{wt} \%]}\end{array}$ & $\begin{array}{c}\text { ICP-MS } \\
{[\mathrm{wt} \%]}\end{array}$ \\
\hline & \multicolumn{3}{|c|}{$\mathrm{c}-\mathrm{mWCNT}$} \\
$\mathrm{C}$ & 96.70 & 76.82 & - & 89.70 & 80.39 & - \\
$\mathrm{H}$ & 0.24 & - & - & 0.51 & - & - \\
$\mathrm{N}$ & 0.76 & 14.61 & - & 0.66 & 11.14 & - \\
$\mathrm{O}$ & 2.30 & 6.12 & - & 9.13 & 8.47 & - \\
$\mathrm{Al}$ & - & 0.55 & 0.15 & - & 0.00 & 0.010 \\
$\mathrm{Fe}$ & - & 1.90 & 0.63 & - & 0.00 & 0.000
\end{tabular}

Thermal processing of nanotubes for FTIR analysis were the following tasks. Firstly, the removal of adsorbed water (bands 3442 and $1635 \mathrm{~cm}^{-1}$ ), and secondly to show degradation of surface groups and the accompanying formation of $\mathrm{CO}_{2}\left(2342 \mathrm{~cm}^{-1}\right)$, and thirdly to determine the degradation temperature of $-\mathrm{COOH}$ groups $\left(1725 \mathrm{~cm}^{-1}\right)$. Figure 2 compares the FTIR spectra of c-MWCNTs and ox-MWCNTs. They show intense bands at wave numbers $c a .3442 \mathrm{~cm}^{-1}$ (surface $-\mathrm{OH}$ groups, $\mathrm{OH}$ moieties in carboxyl groups and chemisorbed water). The shifts in characteristic wave numbers in the direction of lower wave numbers $\left(3157 \mathrm{~cm}^{-1}\right)$ indicate the presence of strong hydrogen bonds between $-\mathrm{OH}$ groups. The bands in the $1750-1400 \mathrm{~cm}^{-1}$ range can be assigned to $\mathrm{C}=\mathrm{O}$ groups in different environments (carboxylic acid, ketone/quinone) and to $\mathrm{C}=\mathrm{C}$ in aromatic rings. The bands in the $1300-950 \mathrm{~cm}^{-1}$ range demonstrate the presence of $\mathrm{C}-\mathrm{O}$ bonds in various chemical environments. From the transmission FTIR spectra of nanotubes - $\mathrm{OH}\left(3157 \mathrm{~cm}^{-1}\right),-\mathrm{COOH}\left(1725 \mathrm{~cm}^{-1}\right)$ groups and other moieties can be identified, which are responsible for the appearance of bands of wave numbers $1573 \mathrm{~cm}^{-1}(\mathrm{C}=\mathrm{C}$ or $\mathrm{C}=\mathrm{O})$ and $\mathrm{C}-\mathrm{O}-\mathrm{C}$ groups (structural oxides, oxygen bridges, etc.) and overlapping bands in the $1200-1000 \mathrm{~cm}^{-1}$ region. As a result of functionalization, the decreasing relative intensity of the $-\mathrm{OH}$ band is assigned to the associated water $\left(1635\right.$ and $\left.3442 \mathrm{~cm}^{-1}\right)$, while the intensity of the band of the carboxyl $(-\mathrm{COOH})$ occurring at $1725 \mathrm{~cm}^{-1}$ increases. Annealing of previously oxidized samples (ox-MWCNTs) in the $25-250{ }^{\circ} \mathrm{C}$ temperature range leads to desorption of $\mathrm{H}_{2} \mathrm{O}$ and $\mathrm{CO}_{2}$. Gaseous products appear as a result of the decomposition of functional groups such as $-\mathrm{COOH},-\mathrm{OH}$ and
$>\mathrm{C}=\mathrm{O}$. Thermal treatment decreases the relative intensity of the $-\mathrm{OH}$ bands (1635 and $3442 \mathrm{~cm}^{-1}$ ) while increasing that of the band at $2342 \mathrm{~cm}^{-1}\left(\mathrm{CO}_{2}\right.$ trapped in $\mathrm{KBr}$ ) (Fig. 3). Heat treatment at higher temperatures $\left(350-900^{\circ} \mathrm{C}\right)$ leads to the destruction of carboxylic groups $-\mathrm{COOH}$ (decrease in $1725 \mathrm{~cm}^{-1}$ band) and their complete disappearance at $800^{\circ} \mathrm{C}$ (Fig. 4).

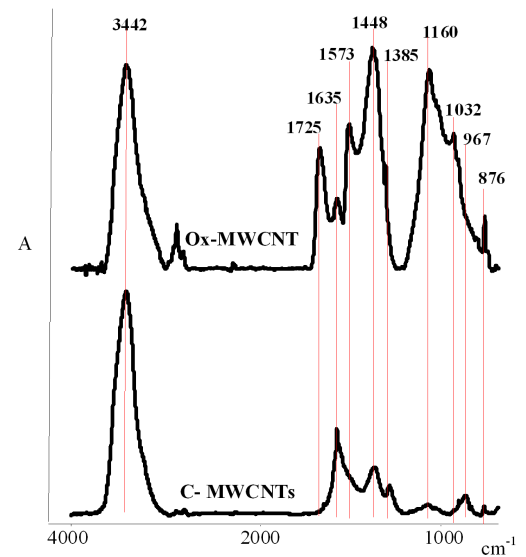

Fig. 2. Comparison of FTIR spectra of c-MWCNTs and ox-MWCNTs.

These observations are confirmed by TG-DTA measurements (Fig. 5). During the heating of samples in the $20-900{ }^{\circ} \mathrm{C}$ temperature range in an oxygen-free atmosphere, rapid mass loss combined with the energy effects in the $200-400{ }^{\circ} \mathrm{C}$ range (water and $\mathrm{CO}_{2}$ removal) and $600-800{ }^{\circ} \mathrm{C}\left(\mathrm{CO}_{2}\right.$ and $\mathrm{CO}$ removal $)$ was observed. The weight loss at $600-800{ }^{\circ} \mathrm{C}$ may be due to the decomposition of carboxylic groups. 


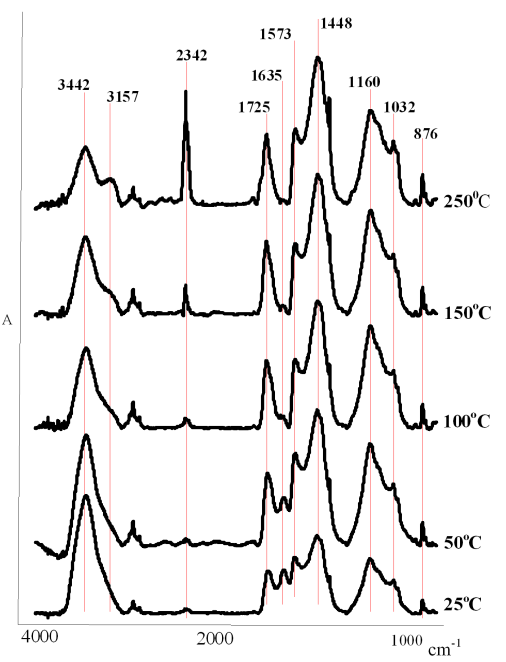

Fig. 3. FTIR spectrum of ox-MWCNTs heated after pressing in the $25-250^{\circ} \mathrm{C}$ range.

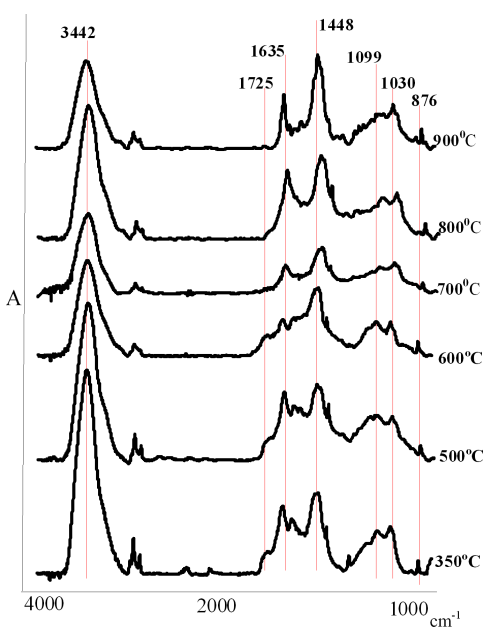

Fig. 4. FTIR spectrum of ox-MWCNTs heated before pressing in the $350-900^{\circ} \mathrm{C}$ range.

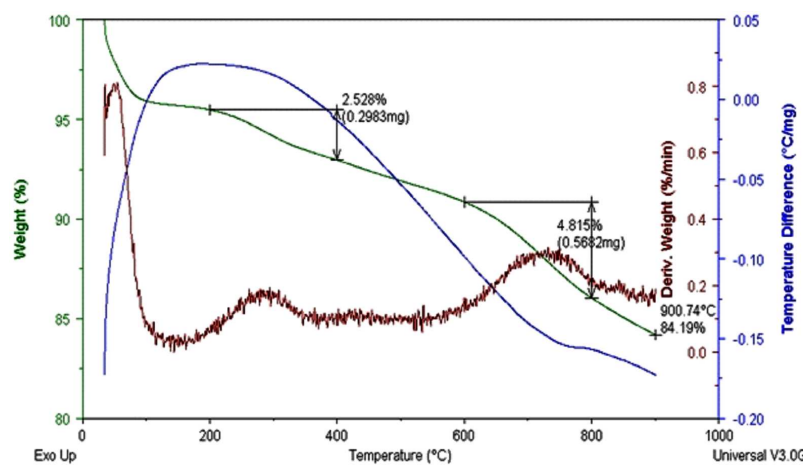

Fig. 5. Thermal analysis of ox-MWCNTs.

\section{Conclusion}

After purification, trace amounts of contaminants were found in the nanotubes: total content of $\mathrm{Fe}$ and $\mathrm{Al}=$ ca. $0.01 \mathrm{wt} \%$; oxygen content $=c a .9 \mathrm{wt} \%$. Electron microscopy images show the structure of tangled carbon nanotubes. The FTIR transmission spectrum allows the identification of the hydroxyl $(-\mathrm{OH})$ and carboxyl $(-\mathrm{COOH})$ groups and others, like $\mathrm{C}=\mathrm{C}, \mathrm{C}=\mathrm{O}$, $\mathrm{C}-\mathrm{O}-\mathrm{C}$. Thermal treatment of the functionalized nanotubes at lower temperatures $\left(25-350^{\circ} \mathrm{C}\right)$ increases the relative content of the $-\mathrm{COOH}$ groups in relation to $-\mathrm{OH}$. Raising the temperature further $\left(350-900^{\circ} \mathrm{C}\right)$ destroys the carboxylic groups - they disappear completely at $800^{\circ} \mathrm{C}$. TGA-DTA gives the CO content, which is approximately $5 \mathrm{wt} \%$.

\section{References}

[1] R.H. Baughman, A.A. Zakhidov, W.A. de Heer, Science 297, 787 (2002).

[2] www.carbonnanotube biz .

[3] Y.P. Sun, K. Fu, Y. Lin, W. Huang, Acc. Chem. Res. 35, 1096 (2002).

[4] J.M. Planeix, N. Coustel, B. Coq, V. Brotons, P.S. Kumbhar, R. Dutartre, P. Geneste, P. Bernier, P.M. Ajayan, J. Am. Chem. Soc. 116, 7935 (1994).

[5] P.G. Collins, P. Avouris, Sci. Am. 283, 62 (2000).

[6] J. Kong, M. Chapline, H. Dai, Adv. Mater. 13, 1384 (2001).

[7] P. Avouris, Acc. Chem. Res. 35, 1026 (2002).

[8] M. Paradise, T. Goswami, Mater Design 28, 1477 (2007).

[9] M.S. Dresselhaus, G. Dresselhaus, P. Avouris, Carbon Nanotubes: Synthesis, Structure, Properties and Applications, Springer-Verlag, New York 2001.

[10] L. Stobinski, J. Mazurkiewicz, H.M. Lin, P. Tomasik, J. Nanosci. Nanotechnol. 5, 2121 (2005).

[11] M. Bonarowska, K.N. Lin, M. Legawiec-Jarzyna, L. Stobinski, W. Juszczyk, Z. Kaszkur, Z. Karpinski, H.M. Lin, Solid State Phenom. 128, 261 (2007).

[12] A.C. Dillon, T. Genneu, K.M. Jones, J.L. Alleman, P.A. Parilla, M.J. Heben, Adv. Mater. 11, 1354 (1999). 